\title{
Autonomous Control and Dynamics Simulation of a 3-Stages Countercurrent Liquid-Liquid Extraction: Trimetozine Purification as a Case Study
}

Zied Hosni, Nazer Rajoub, Ian Houson, Alison Nordon, Brahim Benyahia, Alastair Florence

Submitted date: 17/08/2019 Posted date: 19/08/2019

Licence: CC BY-NC-ND 4.0

Citation information: Hosni, Zied; Rajoub, Nazer; Houson, Ian; Nordon, Alison; Benyahia, Brahim; Florence, Alastair (2019): Autonomous Control and Dynamics Simulation of a 3-Stages Countercurrent Liquid-Liquid Extraction: Trimetozine Purification as a Case Study. ChemRxiv. Preprint.

Liquid-liquid extraction is a technique of extraction heavily used in chemical industry and especially for purification of pharmaceuticals. Trimetozine was used as a case study to test the performance of an implemented platform capable of conducting the extraction of the analyte of interest from its impurities using a washing solvent. Three stages of pairs of mix-er-settler were designed for a full control of the streams flow rates, the temperature, and the speed of stirring. The countercurrent extraction was chosen with an inline IR sensor as a Process Analytical Technology and a Partial Least Squares model to quantify the partitioning of the different analytes in the downstream of the platform. The dynamics of the extraction was followed experimentally and simulated in MATLAB in order to build black-box model.

File list (2)

Liq-liq automated extraction v3.docx (2.42 MiB)

view on ChemRxiv - download file 


\title{
Autonomous control and dynamics simulation of a 3-stages countercurrent liquid-liquid extraction: Trimetozine purification as a case study
}

\author{
Zied Hosni, ${ }^{a}$ Nazer Rajoub, ${ }^{\text {a }}$ Ian Houson, ${ }^{\text {a }}$ Alison Nordon, ${ }^{\mathrm{b}}$ Brahim BenYahia, ${ }^{\mathrm{c}}$ Alastair \\ Florence ${ }^{a}$ \\ ${ }^{a}$ EPSRC Centre for Innovative Manufacturing in Continuous Manufacturing and Crystallisation, \\ Department of Chemical and Process Engineering, University of Strathclyde, James Weir Building, \\ 75 Montrose Street, Glasgow G1 1XJ, United Kingdom \\ ${ }^{b}$ Department of Pure and Applied Chemistry, Centre for Process Analytics and Control Technology \\ (CPACT) and EPSRC CMAC Future Manufacturing Research Hub University of Strathclyde 295 \\ Cathedral Street Glasgow G1 1XL UK \\ ' Loughborough University, Loughborough, United Kingdom
}

KEYWORDS Liquid-liquid extraction, impurities rejection, Aspen simulation, Automation, feedback control

\begin{abstract}
Liquid-liquid extraction is a technique of extraction heavily used in chemical industry and especially for purification of pharmaceuticals. Trimetozine was used as a case study to test the performance of an implemented platform capable of conducting the extraction of the analyte of interest from its impurities using a washing solvent. Three stages of pairs of mixer-settler were designed for a full control of the streams flow rates, the temperature, and the speed of stirring. The countercurrent extraction was chosen with an inline IR sensor as a Process Analytical Technology and a Partial Least Squares model to quantify the partitioning of the different analytes in the downstream of the platform. The dynamics of the extraction was followed experimentally and simulated in MATLAB in order to build black-box model.
\end{abstract}

\section{Introduction}

The conventional workup based on liquid-liquid extraction is a time- and labour-consuming practice. [1]-[3] Dispersive micro-extraction methodologies are pre-treatment techniques based on emulsion formation using solvent-assisted dispersion, kinetic energy, or induction of solvent-oversaturation.[4] The automation of these methods enables improvements in reproducibility and samplethroughput, as well as coupling with modern analytical instrumentation. The miniaturisation of the dispersive liquid-liquid microextraction process is very well achieved when it is coupled with automation.[5], [6] Although certain operations seem difficult to automate and demand intermediate manual intervention such as exchange of solvent, automation has been considered straightforward in many occasions and showed the possibility of incorporating the extraction and the quantification like in the example of the autosampler incorporated with detection systems. Such systems allowed the avoidance of potential contamination, better control of the experimental conditions, a gain in reliability and reproducibility. They enabled also the possibility of performing parallel extractions and increased the throughput of the assay.[1]
Shuur and co-workers reviewed the recent achievements in the field of chiral separation by enantioselective liquid-liquid extraction. It involves host-guest chemistry,[7] extraction and phase transfer mechanisms,[8] and multistage liquid-liquid extraction processing.[9] The authors stated the possible scalability, recyclability and the easy handling of the process based on the enantioselective liq-liq extraction when it is combined with the multistage countercurrent cascade technology.[10]

Salting-out assisted liquid-liquid extraction (SALLE) is a type of homogeneous liquid-liquid extraction that applies the salting-out effect to separate watermiscible organic solvent such as acetonitrile from different matrices. Through 96-well automation, SALLE showed on many occasions that it can be easily integrated into the overall high-throughput LC-MS/MS bioanalysis strategy to increase productivity (as short as $25 \mathrm{~min} /$ plate).[11]

Silvestred et al. stated that, among the techniques for analyte detection in LLE, $67 \%$ of the publications was about the utilisation of UV/visible spectrophotometry,[12] followed by $16 \%$ for atomic 
absorption spectrometry (AAS)[13] and 7\% for spectrofluorometry.[14] It has been also noted that the application of optical detector was compatible with the use of organic solvents. The author highlighted that the variety of the detection techniques are only limited by the presence of trace amounts of one of the immiscible solvents in the other phase. There is also the possibility to incorporate two parallel detectors instead of one detector with two flowthrough cells which enables the simultaneous determination of extractable and non-extractable species.[15]

The ability to more completely automate the traditional liquid-liquid extraction (LLE) technique has been advanced by the microplate format and the use of liquid handling workstations. Liquidliquid extraction procedures can now be performed in a semi-automated mode, freeing analyst time and improving overall productivity. This approach consists of automatically detecting the boundary between the organic and aqueous phases and selectively removes these solvents. The Allex ${ }^{\mathrm{T}}$ (Mettler-Toledo Autochem, Vernon Hills, IL USA) is a good example of a single probe workstation of automated liquid-liquid extraction employing phase boundary sensing technology. It is a fully integrated benchtop system that automates the whole process of extraction and workup. This includes the addition of aqueous and organic solutions, sample mixing via vigorous aspiration and dispensing, phase separation, and phase distribution. The probe, the chambers and the nozzles are appropriately cleaned between the samples. This process can be carried out in either serial or batch mode. The full unit is controlled by the software that combines single action steps into a customised user program. The workstation was designed and marketed for medicinal chemistry application but it is easily applicable for analytical liq-liq extraction in tube or microplate format. Three probe sizes are also available depending on the scale of the experiment of interest. The power of this technology is accentuated by the fact that the two phases can be detected even when the user cannot visually see the interface. There are also error handling tools integrated and used to spot and flag the presence of foaming or an emulsion or to use present height definition. The Allex system can perform up to 60 separations per hour according to its manufacturer.

The Quadro ${ }^{\circledR} 96$ (Tomtec Inc., Hamden, CT USA) is another example of the utilisation of the automation strategy but with the combination of elementary manual steps that were justified by the manufacturer. This latter claimed that this combination of manual and automated processes enhanced the productivity of the instrument. The workstation very fast sample turnaround that is required for a bioanalytical program that supports the drug discovery process in pharmaceutical research.[16]
Wells cited thoroughly many other examples of automated and semi-automated liq-liq extraction systems that are capable to perform the whole process in a very short time especially when multiple autosamplers are incorporated.[17]-[19] Among these examples, Zweigenbaum and Henion reported the high throughput determination of six benzodiazepines in human urine using a selected reaction monitoring LC/MS method with sample preparation using semiautomated LLE. When four autosamplers were connected to one chromatographic column and one tandem mass spectrometer, 1152 samples (twelve 96-well plates) were analysed.[20]

Khajeh reported the utilisation of the Box-Behnken design[21] (a second-order multivariate technique based on three-level incomplete factorial designs) to navigate in the experimental conditions (with a reduced number of experiments $<15$ ) that control the liquid-liquid extraction of lead from food. This design of experiments generated data for the modelling of the response surface. Three independent variables, including $\mathrm{pH}$ of the solution, the concentration of dithizone as a chelating agent and extracting solvent volume were coded at three different levels $(-1,0$ and 1$)$. The Box-Behnken design allowed to get a formula that connects the experimental conditions and their interactions with the extraction efficiency of the process. The multiple regression analysis permitted the obtention of the coefficients of the equation. Finally, the response Surface modelling was employed to determine the optimum conditions of extraction at $\mathrm{pH} 8.4$, the volume of chloroform at $0.45 \mathrm{~mL}$, and the concentration of dithizone at $0.5 \mathrm{ug} \cdot \mathrm{L}^{-1} \cdot[22]$

As a proof of concept for the application of the automated platform on a liquid-liquid extraction process, the synthesis of a commercial sedative was conducted to prepare a mixture of the compound of interest and its impurities that are aimed to be isolated. This reaction required an acid chloride (the trimethoxybenzoyl chloride) and morpholine in the presence of biphasic basic mixture of water/methyl tetrahydrofuran. The main impurity in this case is the hydrolysis of the acid chloride with water which led to the formation of the carboxylic acid (the trimethoxybenzoic acid).

Graph 1. Synthesis of Trimetozine in Me-THF in presence of sodium carbonate.

\section{Experiments}

\subsection{Materials and reagents}

The chemicals employed for the synthesis of trimetozine were obtained from Sigma Aldrich 
(UK). Morpholine was provided by Santa Cruz (US) and the trimethoxybenzoic acid was purchased from Alfa Aeser (UK). Sodium carbonate and butanol were supplied by VWR (UK). HPLC grade acetonitrile, water, methanol were provided by VWR (UK). $\mathrm{D}_{2} \mathrm{O}$ and deuterated chloroform were purchased from Sigma-Aldrich (UK).

\subsection{Trimetozine synthesis}

To a mixture of morpholine (87.1 mg, $1 \mathrm{mmol}$ ) dissolved Me-THF ( $25 \mathrm{~mL}$ ) was added a mixture of sodium carbonate $(48.1 \mathrm{mg}, 2 \mathrm{mmol})$, and the resultant mixture was stirred at room temperature for $15 \mathrm{~min}$ and then trimethoxybenzoyl chloride (230.6mg, $1 \mathrm{mmol}$ ) was added. The mixture was stirred for $48 \mathrm{~h}$. On completion, the solid was filtered with a sintered glass funnel. The filtrate was evaporated on a rotary evaporator, the residue was dried on a two-stage pump (high vacuum) and the resultant product was recrystallized and dried in a desiccator, while the liquid products were purified by vacuum distillation.[23] ${ }^{1} \mathrm{H}$ NMR (300 $\mathrm{MHz}, \mathrm{CDCl}_{3}$ ) $\delta 6.61$ (s, 2H, Ar-H), $3.86(\mathrm{~s}, 6 \mathrm{H}, 2 \times \mathrm{OCH} 3), 3.85$ (s, $3 \mathrm{H}$, $\mathrm{OCH} 3), 3.69(\mathrm{br}, \mathrm{s}, 8 \mathrm{H}, 4 \times \mathrm{CH} 2)$.

\subsection{Fourier transform infrared spectroscopy (FT-IR)}

The infrared spectroscopy data of all starting materials were recorded on a Tensor II FT-IR spectrophotometer (Bruker Optik $\mathrm{GmbH}$ ) over the range of $4000-400 \mathrm{~cm}^{-1}$ by pressed pellet method using diamond. The spectra were acquired by accumulation of 256 scans with $4 \mathrm{~cm}^{-1}$ resolution via Opus 7.5 software. The absorption values were represented in $\mathrm{cm}^{-1}$.

\subsection{Multivariate analysis}

The multivariate analysis was carried out to build a mathematical model capable of predicting the composition of an organic solutions from their corresponding infrared absorption spectra.

\subsubsection{Limit of detection}

The first step to build a robust mathematical mode is to heck the reliability of the used data. Signal to noise ratio is the ratio between the average $\bar{x}$ of each signal and its standard deviation $\bar{\sigma}$. The higher the number of recorded spectra $\mathrm{n}$ is, the higher the signal to noise ratio becomes. This is a crucial factor that determine the accuracy of prediction.

$$
\frac{S}{N}=\frac{\dot{x}}{\dot{\sigma}} \quad \alpha \quad \mathrm{n}^{1 / 2}
$$

The limit of detection was calculated for each of the molecules present in the organic mixtures. This involved the product (Trimetozine) and the impurities (the remaining trimethoxynezoyl chloride, the morpholine and the trimethoxybenzoic acid that was the result of the hydrolysis of the trimethoxybenzoic acid).

\subsubsection{Design of experiments}

The Box-Behnken design of experiments was exploited in this work. It took into account 3 independent variables (three levels of concentration for each of the 3 chemicals). The three levels of concentration selected for this design are in $\mathrm{mg} / \mathrm{ml}$ as follow: 0,5 , and 10 . The set of experiments included 3 centre points. It is noteworthy that the trimethoxybenzoyl chloride was not included in this analysis because it showed a spontaneous reaction with the morpholine which was likely to bias the results of the analysis. The design of experiments was conducted through Modde software.[24] This later generated 15 combinations between the three molecules of interest.

\subsubsection{Partial Least square design (PLS)}

The PLS model script was implemented in LabVIEW.[25], [26] It predicted the ratios between the ingredients of mixtures from unknown IR spectra. MATLAB node in LabVIEW acquired preprocessed data from the spectra and used the PLS model that was built from the dataset of spectra compiled from the Box-Behnken design of experiments.

\subsection{Dynamics monitoring simulation}

and

The pumps were initially calibrated and primed the liquid feeding with the $1200 \mathrm{ml}$ mixture containing $1 \mathrm{mg} / \mathrm{ml}$ trimetozine and $\mathrm{mg} / \mathrm{ml}$ trimethoxybenzoic acid in methyl tetrahydrofuran The flow rate of organic stream was maintained at $2 \mathrm{ml} / \mathrm{min}$ and the aqueous flow rate was triggered after 135 min from the start of the feed flow at $1 \mathrm{ml} / \mathrm{min}$. The stirring in each mixing flask was kept at $400 \mathrm{rpm}$ and $22 \mathrm{C}$. The sampling was conducted every $15 \mathrm{~min}$ for 6 hours. The HPLC analysis was carried out using a reverse phase Poroshell HPLC column. Each sample (ie.50 $\mu \mathrm{l}$ ) from the extract was diluted in950 $\mu$ l of acetonitrile. This necessary to avoid eventual damage to the column and a drop of the chromatographic separation performances. 50 $\mathrm{mM}$ Ammonium formate solution buffered at $\mathrm{pH}$ of 3 was used to wash the column peaks area of both TMZ and BA were directly used to find the dynamic evolution of phase partitions as seen in Graph 4.

\subsection{Automation of 3-stages liquid-liquid extraction}




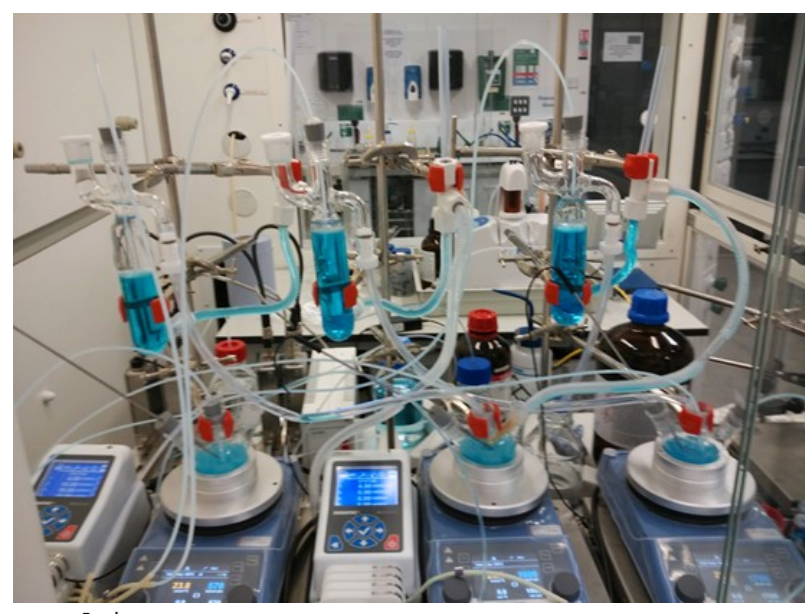

Feed

+ desired solute

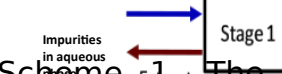

Raffinate (Desired

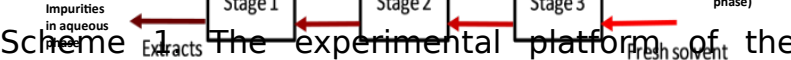
liquid-lic n running with a mixture of blue coloured water and n-butanol showing the four modules of flow monitoring and process control.

The platform designed to conduct the countercurrent liquid-liquid extraction consisted of 4 main modules and a glassware scaffold. The glassware scaffold was based on a triplicate of a mixer (3-necks Morton round bottom flask of $100 \mathrm{ml}$ ) and a cactus settler (a glass cylinder with two arms). Each pair of a mixer and settler form a stage in the platform. The two immiscible solvents were mixed thoroughly in the mixers then separated in the equilibrium in two distinct phases. Each setter had t4 orifices. Two of them were used to link to the vent line and ensure that the volatile organic solvent was purged properly in the fume hood. The last two outlets were employed to transfer the organic phase from the top to the mixer of the following stage and to recycle the aqueous phase to the mixer of the previous stage. There were two exceptions to this portioning: in the first stage, the bottom layer was not recycled but it was collected as the residues that contained most of the impurities. The second exception was in the last stage where the top layer was not transferred to an additional stage but collected as the extractant where the purified Trimetozine was concentrated in methyl tetrahydrofuran. The platform possessed, additionally, tee connectors for each stage to link the settler with the recycling path. These teeshaped connectors were set in an adjustable Hight close to the settler and practically slightly above the level of liquid in the settler. This part had a crucial role in the system as it defined the amount of liquid that was substrate from the two phases in the settlers. The first module was the Reglo module that controlled the peristaltic pumps. This later was Ismatec Reglo pumps. They possessed 4 independent channels. MHLL and Vyton tubing were used when water and butanol were employed as solvents for the platform. Gore StaPure PHL material was employed for the peristaltic tubing when methyl tetrahydrofuran was the solvent in the organic phase. The flow rate could be controlled in both direction and could be varied between 0.001 and $23 \mathrm{ml} / \mathrm{min}$. The inner diameter of the flexible tubing was set always to $3.2 \mathrm{~mm}$. Before the start of the extraction, all the channels were calibrated individually with water to adjust the speed of motor rotation according to the desired flow rate. The pumps controlled the flow rates of the feed that contained the Trimetozine and the different impurities, water that is flowing in countercurrent starting from the last stage of the system. Three additional channels ensured the transfer of the liquid between the mixer and the settlers. The internal flow was set always higher than the sum of the flow rates for the feet and the extractant (water).

The second module was the Bronkhorst flow meters that ensured the measurement of the flow rates of the liquid feeding the first mixer $d$ the last mixer and the liquid moving between the mixers and the settlers in the difference $t$ stages. The flowmeters were remotely monitored through a dedicated software provided from their supplier and integrated into LabVIEW.

The third module was the IKA module. It enables the control of the stirring speed and the temperature in the mixers and monitors the mass of the liquid in the same mixers. Each stage possesses a hotplate that was remotely controlled with the PC. The bilateral connection enabled sending the user commands to control the temperature and the speed of stirring. Simultaneously the hot plate sent a feed back to the PC about the actual measurement of these experimental parameters via a thermocouple and a magnetic sensor for the temperature and the speed of stirring, respectively.

The last module was the react IR module. It consisted of a portable ReactIR MCT instrument with an ATR dip probe. This later was placed inside the settler of the first stage to monitor the composition of the organic phase and to spot eventual perturbation. The frequency of IR spectra acquisition was set at 1 spectrum every $10 \mathrm{~min}$. The recorded spectra were retrieved by LabVIEW and the PLS predictive model was exploited to estimate the composition of the organic phase in terms of Trimetozine and the corresponding impurities 


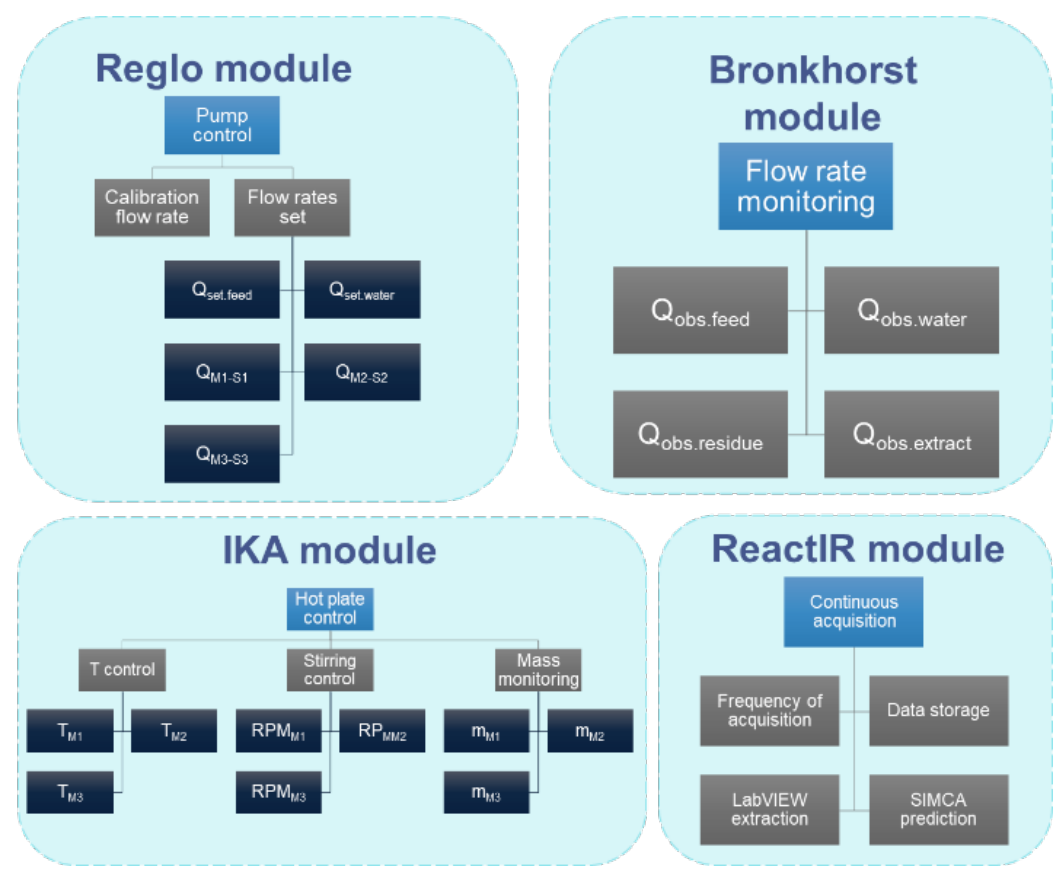

Graph 2. Four integrated modules for the automation of the 3-stages countercurrent liquid-liquid extraction.

3.

\section{Results and discussion}

\subsection{PLS predictive performance for PAT}

The Partial Least Squares method was exploited to build a predictive model that is able to convert the IR spectra acquired by the PAT implemented in the automated platform. This step is vital for any further feedback loop of the automated extraction. It allowed to covert a graphical analytical spectrum to a quantitative monitoring of the concentration of the different ingredients at the downstream of the process. It is clear from Graph 3 that the PLS model performs optimally by including 3 PLS components. The variance explained by the generated reached $96 \%$ and the mean square error dropped down to 4 as illustrated graph 3.B.

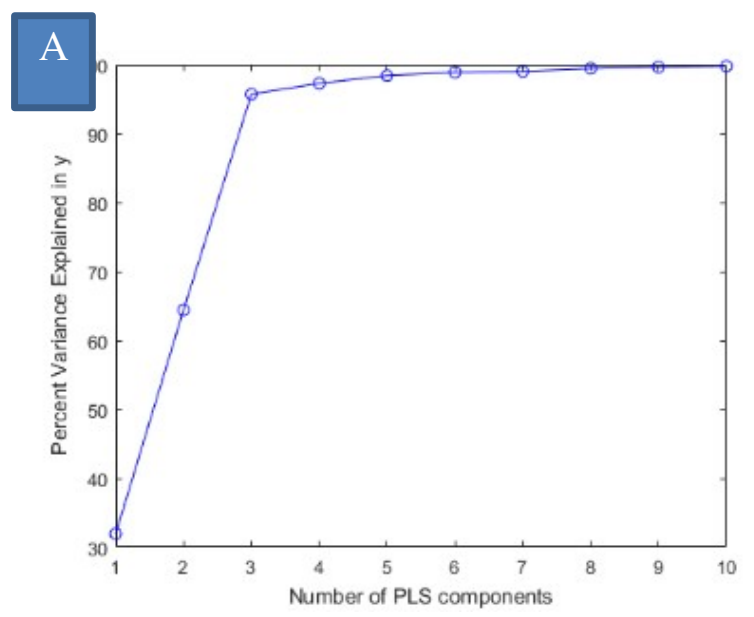

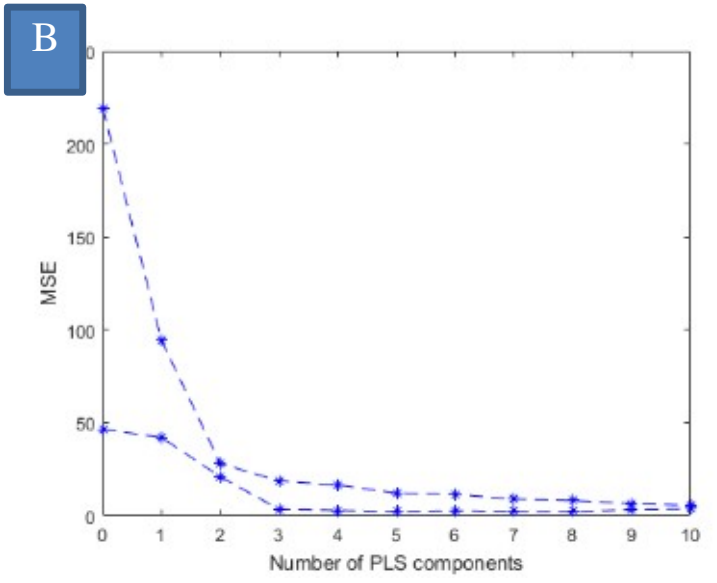

Graph 3: Predictive performance of the PLS model as a function of the number of considered components. A) Percentage of accumulated variance explained by the model. B) Mean square error.

\subsection{Dynamics of the automated 3- stages LLE platform}

The elucidation of the dynamics that drive the extraction in the 3-stage countercurrent liq-liq extract is important to conduct any further optimisation of the extraction factors in order to improve the purity of the product or to increase the yield of extract or to minimise the energy and the water required to carry out the extraction. The dynamics of the extraction was monitored by analysing periodically samples extracted from the downstream of the platform via HPLC separation and quantification. The Graph 4 shows the partitioning of trimetozine and the trimethoxybenzoic acid in extract and in the 
residue. Therefore, it was straightforward to evaluate the variation of these two analytes over time in both extract and residue. The platform achieved a steady state (i.e. A stationary phase illustrated by a plateau in the concentration of the

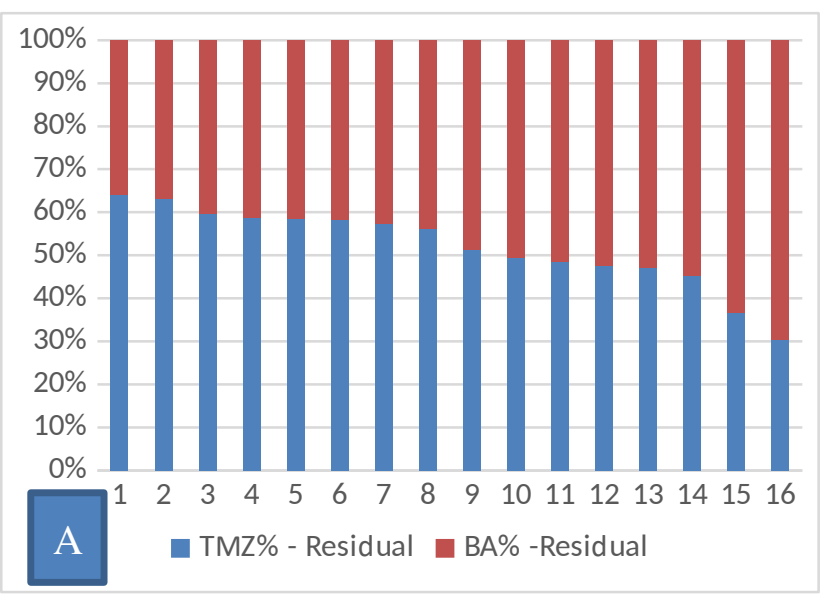

analytes). As shown in Graph 4.B, the extraction platform reached the steady state from the $10^{\text {th }}$ sample which is occurring after $270 \mathrm{~min}$ from the start of the extraction.

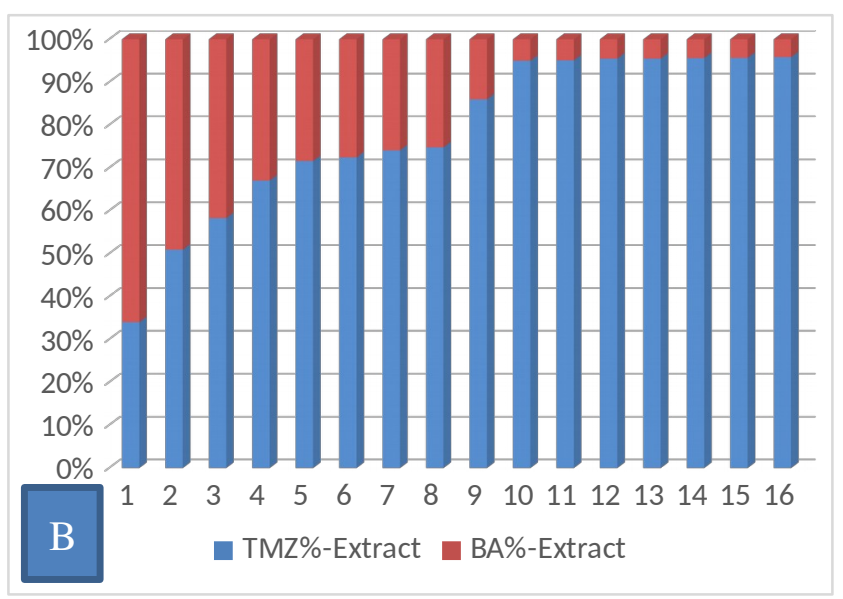

Graph4: Percentage of extraction over time in the 3-stages liquid-liquid extraction platform. A) HPLC percentage of trimetozine and trimethoxybenzoic acid in the residue. B). HPLC percentage of trimetozine and trimethoxybenzoic acid in the extract.
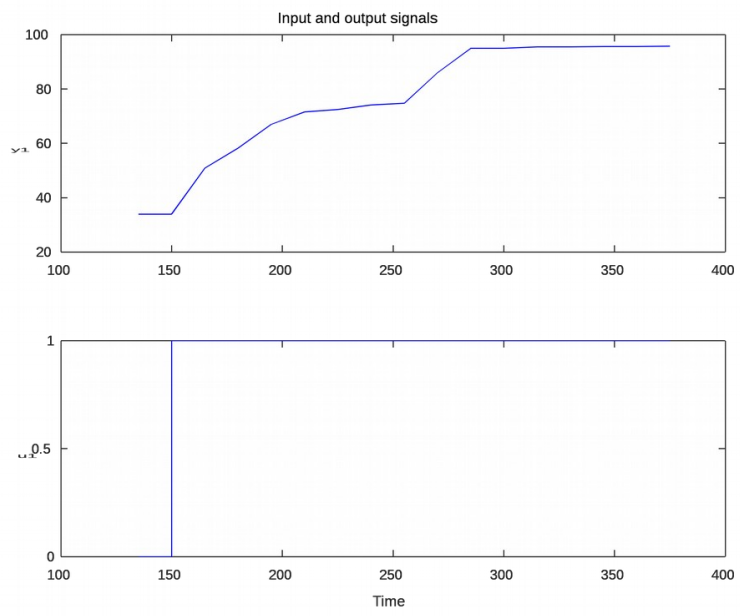

Graph 5: The dynamics of extraction over time. Y1 represents the yield of extraction and $t 1$ represents the flow rate of water in the platform.

The addition of water was triggered after 135 min from the beginning of extraction. The following sampling demonstrated the behaviour of the platform in terms of trimetozine extraction process. Water flow rate was stabilised at $1 \mathrm{ml} / \mathrm{min}$ over the whole process. The concentration of trimetozine kept increasing until achieving $95.04 \%$ after $285 \mathrm{~min}$ from the starting of the liquid flow within the platform.

The dynamics of extraction monitored in the liq-liq extraction platform was simulated using Simulink in MATLAB. Different regression models were generated in order to fit the experimental behaviour of extraction. The blue curve represents the initial fitting and the $5^{\text {th }}$ regression in pink represent the best fitting achieved by optimising the regression coefficients. Therefore, at tf5, $92.19 \%$ of fitting was achieved at tf5 to predict the dynamics of the extraction platform before reaching the steady-state.

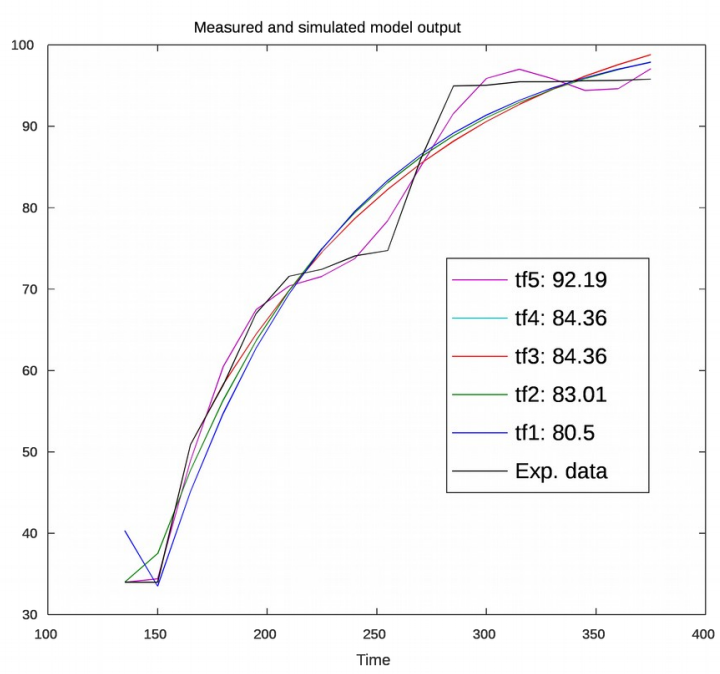

Graph 6: Five different models gathered from the black-box simulation of the extraction dynamics.

\section{Conclusions}


A 3-stages liq-liq counter current extraction platform was implemented to conduct the extraction of trimetozine from a mixture containing mainly analyte of interest and the impurity (trimethoxybenzoic acid). The platform controlled and monitored the flow rates at the different streams of the platform and the PAT module allowed the monitoring of the extraction performance through an inline IR probe. A full evaluation of the partitioning of the analyte of interest was conducted by exploiting a PLS predictive model. The obtained concentrations of the extract and the impurity were employed to monitor the dynamics of the extraction and to build a black-box model to predict the extraction behaviour of trimetozine before reaching a steady-state regime.

\section{AUTHOR INFORMATION}

\section{Corresponding Author}

* (Word Style

"FA_Corresponding_Author_Footnote"). Give contact information for the author(s) to whom correspondence should be addressed.

\section{Present Addresses}

tIf an author's address is different than the one given in the affiliation line, this information may be included here.

\section{Author Contributions}

The manuscript was written through contributions of all authors. / All authors have given approval to the final version of the manuscript. / $\ddagger$ These authors contributed equally. (match statement to author names with a symbol)

\section{Funding Sources}

Any funds used to support the research of the manuscript should be placed here (per journal style).

\section{Notes}

Any additional relevant notes should be placed here.

\section{ACKNOWLEDGMENT}

(Word Style "TD_Acknowledgments"). Generally, the last paragraph of the paper is the place to acknowledge people (dedications), places, and financing (you may state grant numbers and sponsors here). Follow the journal's guidelines on what to include in the Acknowledgement section.

\section{ABBREVIATIONS}

CCR2, CC chemokine receptor 2; CCL2, CC chemokine ligand 2; CCR5, CC chemokine receptor 5; TLC, thin layer chromatography.

\section{REFERENCES}

[1] M. Alexovič, B. Horstkotte, I. Šrámková, P. Solich, and I. Sabo, "Automation of dispersive liquid-liquid microextraction and related techniques. Approaches based on flow, batch, flow-batch and in-syringe modes," TrAC Trends Anal. Chem., vol. 86, pp. 39-55, Jan. 2017.

[2] N. Weeranoppanant et al., "Design of Multistage Counter-Current Liquid-Liquid Extraction for Small-Scale Applications," Ind. Eng. Chem. Res., 2017.

[3] E. Boyaci et al., "Sample preparation with solid phase microextraction and exhaustive extraction approaches: Comparison for challenging cases," Analytica Chimica Acta. 2015.

[4] R. Jain and R. Singh, "Applications of dispersive liquidliquid micro-extraction in forensic toxicology," TrAC . Trends in Analytical Chemistry. 2016.

M. Saraji and M. K. Boroujeni, "Recent developments in dispersive liquid-liquid microextraction," Anal. Bioanal. Chem., 2013.

P. L. Kole, G. Venkatesh, J. Kotecha, and R. Sheshala, "Recent advances in sample preparation techniques for effective bioanalytical methods," Biomedical Chromatography. 2011.

E. Binxterhuis, J. B. Gualtierotti, S. J. Wezenberg J G. de Vries, and B. L. Feringa, "Highly Efficient and Robust Enantioselective Liquid-Liquid Extraction of 1,2-Amino Alcohols utilizing VAPOL- and VANOL-based Phosphoric Acid Hosts," ChemSusChem, 2018.

[8] B. Schuur, B. J. V. Verkuijl, A. J. Minnaard, J. G. de Vries, H. J. Heeres, and B. L. Feringa, "Chiral separation by enantioselective liquid-liquid extraction," Org. Biomol. Chem., vol. 9, no. 1, pp. 36-51, 2011.

[9] K. Tang, Y. Wang, P. Zhang, Y. Huang, and G. Dai, "Process optimization of continuous liquid-liquid extraction in centrifugal contactor separators for separation of oxybutynin enantiomers," Sep. Purif. Technol., 2015.

[10] B. Schuur, B. J. V Verkuijl, A. J. Minnaard, J. G. de Vries, H. J. Heeres, and B. L. Feringa, "Chiral separation by enantioselective liquid-liquid extraction," Org. Biomol. Chem., vol. 9, no. 1, pp. 36-51, 2011.

[11] Y. Q. Tang and N. Weng, "Salting-out assisted liquidliquid extraction for bioanalysis.," Bioanalysis, vol. 5, no. 12 , pp. 1583-98, 2013

[12] B. Peng, Y. Shen, Z. Gao, M. Zhou, Y. Ma, and S. Zhao, "Determination of total iron in water and foods by dispersive liquid-liquid microextraction coupled with microvolume UV-vis spectrophotometry," Food Chem. 2015

[13] M. Tuzen and O. Z. Pekiner, "Ultrasound-assisted ionic liquid dispersive liquid-liquid microextraction combined with graphite furnace atomic absorption spectrometric for selenium speciation in foods and beverages," Food Chem., 2015.

[14] W. Ahmad, A. Al-Sibaai, A. S. Bashammakh, H. Alwael, and M. S. El-Shahawi, "An ultrasound-assisted ion association dispersive liquid-liquid microextraction coupled with micro-volume spectrofluorimetry for chromium speciation," RSC Adv., 2016.

[15] C. I. C. Silvestre, J. L. M. Santos, J. L. F. C. Lima, and E. A. G. Zagatto, "Liquid-liquid extraction in flow analysis: A critical review," Anal. Chim. Acta, vol. 652, no. 1-2, pp. 54-65, Oct. 2009.

[16] "Chapter 10 Liquid-liquid extraction: Automation strategies," in Progress in Pharmaceutical and Biomedical Analysis, vol. 5, no. C, 2003, pp. 327-360. 
[17] F. Maya, J. M. Estela, and V. Cerdà, "Completely automated in-syringe dispersive liquid-liquid microextraction using solvents lighter than water," Anal. Bioanal. Chem., vol. 402, no. 3, pp. 1383-1388, Jan. 2012.

[18] C. S. M. Liew, X. Li, H. Zhang, and H. K. Lee, "A fully automated analytical platform integrating water sampling-miniscale-liquid-liquid extraction-full evaporation dynamic headspace concentration-gas chromatography-mass spectrometry for the analysis of ultraviolet filters," Anal. Chim. Acta, 2018.

[19] I. Domínguez, R. Romero González, F. J. Arrebola Liébanas, J. L. Martínez Vidal, and A. Garrido Frenich, "Automated and semi-automated extraction methods for GC-MS determination of pesticides in environmental samples," Trends in Environmental Analytical Chemistry. 2016.

[20] J. Zweigenbaum, K. Heinig, S. Steinborner, T. Wachs, and J. Henion, "High-Throughput Bioanalytical LC/MS/MS Determination of Benzodiazepines in Human Urine: 1000 Samples per 12 Hours," Anal. Chem., vol. 71, no. 13, pp. 2294-2300, 1999.

[21] S. L. C. Ferreira et al., "Box-Behnken design: An alternative for the optimization of analytical methods,"
Analytica Chimica Acta. 2007.

[22] M. Khajeh, "Response surface modelling of lead preconcentration from food samples by miniaturised homogenous liquid-liquid solvent extraction: BoxBehnken design," Food Chem., vol. 129, no. 4, pp. 1832-1838, 2011.

[23] K. M. Khan et al., "Leishmanicidal potential of Nsubstituted morpholine derivatives: Synthesis and structure-activity relationships," Nat. Prod. Res., vol. 23, no. 5, pp. 479-484, 2009.

[24] I. Schmid and J. Aschoff, "A scalable software framework for data integration in bioprocess development," Eng. Life Sci., 2017.

[25] N. Hernández, R. Kiralj, M. M. C. Ferreira, and I. Talavera, "Critical comparative analysis, validation and interpretation of SVM and PLS regression models in a QSAR study on HIV-1 protease inhibitors," Chemom. Intell. Lab. Syst., vol. 98, no. 1, pp. 65-77, 2009.

[26] J. H. Moore, "Artificial intelligence programming with LabVIEW: genetic algorithms for instrumentation control and optimization," Comput. Methods Programs Biomed., vol. 47, no. 1, pp. 73-79, 1995. 


\title{
Autonomous control and dynamics simulation of a 3-stages counter- current liquid-liquid extraction: Trimetozine purification as a case study
}

\author{
Zied Hosni, ${ }^{\mathrm{a}}$ Nazer Rajoub, ${ }^{\mathrm{a}}$ Ian Houson, ${ }^{\mathrm{a}}$ Alison Nordon, ${ }^{\mathrm{b}}$ Brahim BenYahia, ${ }^{\mathrm{c}}$ Alastair Florence ${ }^{\mathrm{a}}$ \\ ${ }^{a}$ EPSRC Centre for Innovative Manufacturing in Continuous Manufacturing and Crystallisation, Department of Chemical \\ and Process Engineering, University of Strathclyde, James Weir Building, 75 Montrose Street, Glasgow G1 1XJ, United \\ Kingdom \\ ${ }^{b}$ Department of Pure and Applied Chemistry, Centre for Process Analytics and Control Technology (CPACT) and EPSRC \\ CMAC Future Manufacturing Research Hub University of Strathclyde 295 Cathedral Street Glasgow G1 1XL UK \\ ${ }^{c}$ Loughborough University, Loughborough, United Kingdom
}

\section{KEYWORDS Liquid-liquid extraction, impurities rejection, Aspen simulation, Automation, feedback control}

\begin{abstract}
Liquid-liquid extraction is a technique of extraction heavily used in chemical industry and especially for purification of pharmaceuticals. Trimetozine was used as a case study to test the performance of an implemented platform capable of conducting the extraction of the analyte of interest from its impurities using a washing solvent. Three stages of pairs of mixer-settler were designed for a full control of the streams flow rates, the temperature, and the speed of stirring. The countercurrent extraction was chosen with an inline IR sensor as a Process Analytical Technology and a Partial Least Squares model to quantify the partitioning of the different analytes in the downstream of the platform. The dynamics of the extraction was followed experimentally and simulated in MATLAB in order to build black-box model.
\end{abstract}

\section{Introduction}

The conventional workup based on liquid-liquid extraction is a time- and labour-consuming practice.[1]-[3] Dispersive micro-extraction methodologies are pre-treatment techniques based on emulsion formation using solvent-assisted dispersion, kinetic energy, or induction of solvent-oversaturation.[4] The automation of these methods enables improvements in reproducibility and sample-throughput, as well as coupling with modern analytical instrumentation. The miniaturisation of the dispersive liquid-liquid microextraction process is very well achieved when it is coupled with automation. [5], [6] Although certain operations seem difficult to automate and demand intermediate manual intervention such as exchange of solvent, automation has been considered straightforward in many occasions and showed the possibility of incorporating the extraction and the quantification like in the example of the autosampler incorporated with detection systems. Such systems allowed the avoidance of potential contamination, better control of the experimental conditions, a gain in reliability and reproducibility. They enabled also the possibility of performing parallel extractions and increased the throughput of the assay.[1]

Shuur and co-workers reviewed the recent achievements in the field of chiral separation by enantioselective liquid-liquid extraction. It involves host-guest chemistry,[7] extraction and phase transfer mechanisms, [8] and multistage liquid-liquid extraction processing.[9] The authors stated the possible scalability, recyclability and the easy handling of the process based on the enantioselective liq-liq extraction when it is combined with the multistage countercurrent cascade technology.[10]

Salting-out assisted liquid-liquid extraction (SALLE) is a type of homogeneous liquid-liquid extraction that applies the salting-out effect to separate water-miscible organic solvent such as acetonitrile from different matrices. Through 96-well automation, SALLE showed on many occasions that it can be easily integrated into the overall high-throughput LC-MS/MS bioanalysis strategy to increase productivity (as short as $25 \mathrm{~min} /$ plate).[11]

Silvestred et al. stated that, among the techniques for analyte detection in LLE, $67 \%$ of the publications was about the utilisation of UV/visible spectrophotometry,[12] followed by $16 \%$ for atomic absorption spectrometry (AAS)[13] and 7\% for spectrofluorometry.[14] It has been also noted that the application of optical detector was compatible with the use of organic solvents. The author highlighted that the variety of the detection techniques are only limited by the presence of trace amounts of one of the immiscible solvents in the other phase. There is also the possibility to incorporate two parallel detectors instead of one detector with two flowthrough cells which enables the simultaneous determination of extractable and non-extractable species.[15]

The ability to more completely automate the traditional liquid-liquid extraction (LLE) technique has been advanced by the microplate format and the use of liquid handling workstations. Liquidliquid extraction procedures can now be performed in a semi-automated mode, freeing analyst time and improving overall productivity. This approach consists of automatically detecting the boundary between the organic and aqueous phases and selectively removes these solvents. The Allex TM (Mettler-Toledo Autochem, Vernon Hills, IL USA) is a good example of a single probe workstation of automated liquid-liquid extraction employing phase boundary sensing technology. It is a fully integrated benchtop system that automates the whole process of extraction and workup. This includes the addition of aqueous and organic solutions, sample mixing via vigorous aspiration and dispensing, phase separation, and 
phase distribution. The probe, the chambers and the nozzles are appropriately cleaned between the samples. This process can be carried out in either serial or batch mode. The full unit is controlled by the software that combines single action steps into a customised user program. The workstation was designed and marketed for medicinal chemistry application but it is easily applicable for analytical liq-liq extraction in tube or microplate format. Three probe sizes are also available depending on the scale of the experiment of interest. The power of this technology is accentuated by the fact that the two phases can be detected even when the user cannot visually see the interface. There are also error handling tools integrated and used to spot and flag the presence of foaming or an emulsion or to use present height definition. The Allex system can perform up to 60 separations per hour according to its manufacturer.

The Quadro ${ }^{\circledR} 96$ (Tomtec Inc., Hamden, CT USA) is another example of the utilisation of the automation strategy but with the combination of elementary manual steps that were justified by the manufacturer. This latter claimed that this combination of manual and automated processes enhanced the productivity of the instrument. The workstation very fast sample turnaround that is required for a bioanalytical program that supports the drug discovery process in pharmaceutical research.[16]

Wells cited thoroughly many other examples of automated and semi-automated liq-liq extraction systems that are capable to perform the whole process in a very short time especially when multiple autosamplers are incorporated.[17]-[19] Among these examples, Zweigenbaum and Henion reported the high throughput determination of six benzodiazepines in human urine using a selected reaction monitoring LC/MS method with sample preparation using semi-automated LLE. When four autosamplers were connected to one chromatographic column and one tandem mass spectrometer, 1152 samples (twelve 96-well plates) were analysed.[20]

Khajeh reported the utilisation of the Box-Behnken design[21] (a second-order multivariate technique based on three-level incomplete factorial designs) to navigate in the experimental conditions (with a reduced number of experiments $<15$ ) that control the liquidliquid extraction of lead from food. This design of experiments generated data for the modelling of the response surface. Three independent variables, including $\mathrm{pH}$ of the solution, the concentration of dithizone as a chelating agent and extracting solvent volume were coded at three different levels (-1, 0 and 1$)$. The Box-Behnken design allowed to get a formula that connects the experimental conditions and their interactions with the extraction efficiency of the process. The multiple regression analysis permitted the obtention of the coefficients of the equation. Finally, the response Surface modelling was employed to determine the optimum conditions of

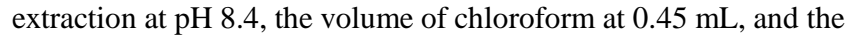
concentration of dithizone at $0.5 \mathrm{ug} . \mathrm{L}^{-1} \cdot[22]$

As a proof of concept for the application of the automated platform on a liquid-liquid extraction process, the synthesis of a commercial sedative was conducted to prepare a mixture of the compound of interest and its impurities that are aimed to be isolated. This reaction required an acid chloride (the trimethoxybenzoyl chloride) and morpholine in the presence of biphasic basic mixture of water/methyl tetrahydrofuran. The main impurity in this case is the hydrolysis of the acid chloride with water which led to the formation of the carboxylic acid (the trimethoxybenzoic acid).

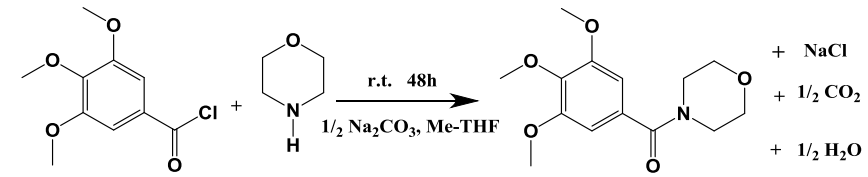

Graph 1. Synthesis of Trimetozine in Me-THF in presence of sodium carbonate.

\section{Experiments}

\subsection{Materials and reagents}

The chemicals employed for the synthesis of trimetozine were obtained from Sigma Aldrich (UK). Morpholine was provided by Santa Cruz (US) and the trimethoxybenzoic acid was purchased from Alfa Aeser (UK). Sodium carbonate and butanol were supplied by VWR (UK). HPLC grade acetonitrile, water, methanol were provided by VWR (UK). $\mathrm{D}_{2} \mathrm{O}$ and deuterated chloroform were purchased from Sigma-Aldrich (UK).

\subsection{Trimetozine synthesis}

To a mixture of morpholine $(87.1 \mathrm{mg}, 1 \mathrm{mmol})$ dissolved Me-THF $(25 \mathrm{~mL})$ was added a mixture of sodium carbonate $(48.1 \mathrm{mg}, 2$ $\mathrm{mmol}$ ), and the resultant mixture was stirred at room temperature for $15 \mathrm{~min}$ and then trimethoxybenzoyl chloride $(230.6 \mathrm{mg}, 1$ mmol) was added. The mixture was stirred for $48 \mathrm{~h}$. On completion, the solid was filtered with a sintered glass funnel. The filtrate was evaporated on a rotary evaporator, the residue was dried on a twostage pump (high vacuum) and the resultant product was recrystallized and dried in a desiccator, while the liquid products were purified by vacuum distillation. [23] ${ }^{1} \mathrm{H} \mathrm{NMR}\left(300 \mathrm{MHz}, \mathrm{CDCl}_{3}\right) \delta 6.61$ (s, 2H, Ar-H), 3.86 (s, 6H, 2×OCH3), 3.85 (s, 3H, OCH3), 3.69 (br, $\mathrm{s}, 8 \mathrm{H}, 4 \times \mathrm{CH} 2)$.

\subsection{Fourier transform infrared spectroscopy (FT-IR)}

The infrared spectroscopy data of all starting materials were recorded on a Tensor II FT-IR spectrophotometer (Bruker Optik $\mathrm{GmbH}$ ) over the range of $4000-400 \mathrm{~cm}^{-1}$ by pressed pellet method using diamond. The spectra were acquired by accumulation of 256 scans with $4 \mathrm{~cm}^{-1}$ resolution via Opus 7.5 software. The absorption values were represented in $\mathrm{cm}^{-1}$.

\subsection{Multivariate analysis}

The multivariate analysis was carried out to build a mathematical model capable of predicting the composition of an organic solutions from their corresponding infrared absorption spectra.

\subsubsection{Limit of detection}

The first step to build a robust mathematical mode is to heck the reliability of the used data. Signal to noise ratio is the ratio between the average $x^{-}$of each signal and its standard deviation $\sigma^{-}$. The higher the number of recorded spectra $n$ is, the higher the signal to noise ratio becomes. This is a crucial factor that determine the accuracy of prediction.

$$
\frac{S}{N}=\frac{\bar{x}}{\bar{\sigma}} \alpha n^{1 / 2}
$$

The limit of detection was calculated for each of the molecules present in the organic mixtures. This involved the product (Trime- 
tozine) and the impurities (the remaining trimethoxynezoyl chloride, the morpholine and the trimethoxybenzoic acid that was the result of the hydrolysis of the trimethoxybenzoic acid).

\subsubsection{Design of experiments}

The Box-Behnken design of experiments was exploited in this work. It took into account 3 independent variables (three levels of concentration for each of the 3 chemicals). The three levels of concentration selected for this design are in $\mathrm{mg} / \mathrm{ml}$ as follow: 0,5 , and 10 . The set of experiments included 3 centre points. It is noteworthy that the trimethoxybenzoyl chloride was not included in this analysis because it showed a spontaneous reaction with the morpholine which was likely to bias the results of the analysis. The design of experiments was conducted through Modde software.[24] This later generated 15 combinations between the three molecules of interest.

\subsubsection{Partial Least square design (PLS)}

The PLS model script was implemented in LabVIEW.[25], [26] It predicted the ratios between the ingredients of mixtures from unknown IR spectra. MATLAB node in LabVIEW acquired pre-processed data from the spectra and used the PLS model that was built from the dataset of spectra compiled from the Box-Behnken design of experiments.

\subsection{Dynamics monitoring and simulation}

The pumps were initially calibrated and primed the liquid feeding with the $1200 \mathrm{ml}$ mixture containing $1 \mathrm{mg} / \mathrm{ml}$ trimetozine and $\mathrm{mg} / \mathrm{ml}$ trimethoxybenzoic acid in methyl tetrahydrofuran The flow rate of organic stream was maintained at $2 \mathrm{ml} / \mathrm{min}$ and the aqueous flow rate was triggered after $135 \mathrm{~min}$ from the start of the feed flow at $1 \mathrm{ml} / \mathrm{min}$. The stirring in each mixing flask was kept at $400 \mathrm{rpm}$ and $22 \mathrm{C}$. The sampling was conducted every $15 \mathrm{~min}$ for 6 hours. The HPLC analysis was carried out using a reverse phase Poroshell HPLC column. Each sample (ie. $50 \mu \mathrm{l}$ ) from the extract was diluted in $950 \mu \mathrm{l}$ of acetonitrile. This necessary to avoid eventual damage to the column and a drop of the chromatographic separation performances. $50 \mathrm{mM}$ Ammonium formate solution buffered at $\mathrm{pH}$ of 3 was used to wash the column peaks area of both TMZ and BA were directly used to find the dynamic evolution of phase partitions as seen in Graph 4.

\subsection{Automation of 3-stages liquid-liquid extraction}

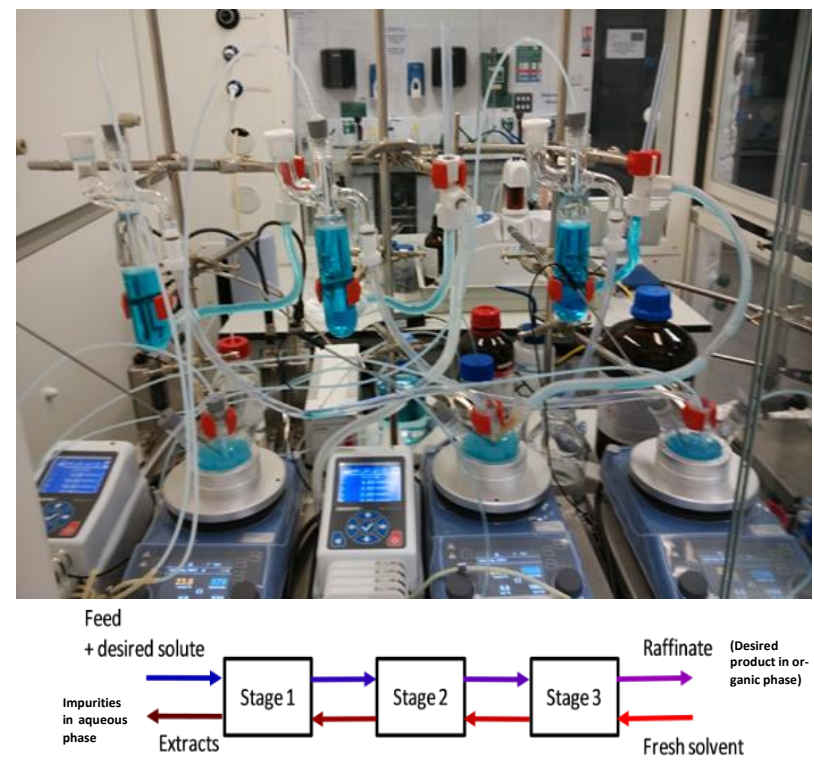

Scheme 1. The experimental platform of the liquid-liquid extraction running with a mixture of blue coloured water and n-butanol showing the four modules of flow monitoring and process control.

The platform designed to conduct the countercurrent liquid-liquid extraction consisted of 4 main modules and a glassware scaffold. The glassware scaffold was based on a triplicate of a mixer (3necks Morton round bottom flask of $100 \mathrm{ml}$ ) and a cactus settler (a glass cylinder with two arms). Each pair of a mixer and settler form a stage in the platform. The two immiscible solvents were mixed thoroughly in the mixers then separated in the equilibrium in two distinct phases. Each setter had 4 orifices. Two of them were used to link to the vent line and ensure that the volatile organic solvent was purged properly in the fume hood. The last two outlets were employed to transfer the organic phase from the top to the mixer of the following stage and to recycle the aqueous phase to the mixer of the previous stage. There were two exceptions to this portioning: in the first stage, the bottom layer was not recycled but it was collected as the residues that contained most of the impurities. The second exception was in the last stage where the top layer was not transferred to an additional stage but collected as the extractant where the purified Trimetozine was concentrated in methyl tetrahydrofuran. The platform possessed, additionally, tee connectors for each stage to link the settler with the recycling path. These teeshaped connectors were set in an adjustable Hight close to the settler and practically slightly above the level of liquid in the settler. This part had a crucial role in the system as it defined the amount of liquid that was substrate from the two phases in the settlers. The first module was the Reglo module that controlled the peristaltic pumps. This later was Ismatec Reglo pumps. They possessed 4 independent channels. MHLL and Vyton tubing were used when water and butanol were employed as solvents for the platform. Gore Sta-Pure PHL material was employed for the peristaltic tubing when methyl tetrahydrofuran was the solvent in the organic phase. The flow rate could be controlled in both direction and could be varied between 0.001 and $23 \mathrm{ml} / \mathrm{min}$. The inner diameter of the flexible tubing was set always to $3.2 \mathrm{~mm}$. Before the start of the extraction, all the channels were calibrated individually with water to adjust the speed of motor rotation according to the desired flow rate. The pumps controlled the flow rates of the feed that contained the Trimetozine and the different impurities, water that is flowing in countercurrent starting from the last stage of the system. Three additional channels ensured the transfer of the liquid between the mixer and the settlers. The internal flow was set always higher than the sum of the flow rates for the feet and the extractant (water).

The second module was the Bronkhorst flow meters that ensured the measurement of the flow rates of the liquid feeding the first mixer $d$ the last mixer and the liquid moving between the mixers and the settlers in the difference $t$ stages. The flowmeters were remotely monitored through a dedicated software provided from their supplier and integrated into LabVIEW.

The third module was the IKA module. It enables the control of the stirring speed and the temperature in the mixers and monitors the mass of the liquid in the same mixers. Each stage possesses a hotplate that was remotely controlled with the PC. The bilateral connection enabled sending the user commands to control the temperature and the speed of stirring. Simultaneously the hot plate sent a feed back to the PC about the actual measurement of these experimental parameters via a thermocouple and a magnetic sensor for the temperature and the speed of stirring, respectively.

The last module was the react IR module. It consisted of a portable ReactIR MCT instrument with an ATR dip probe. This later was placed inside the settler of the first stage to monitor the composition 
of the organic phase and to spot eventual perturbation. The frequency of IR spectra acquisition was set at 1 spectrum every 10 min. The recorded spectra were retrieved by LabVIEW and the PLS predictive model was exploited to estimate the composition of the organic phase in terms of Trimetozine and the corresponding impurities

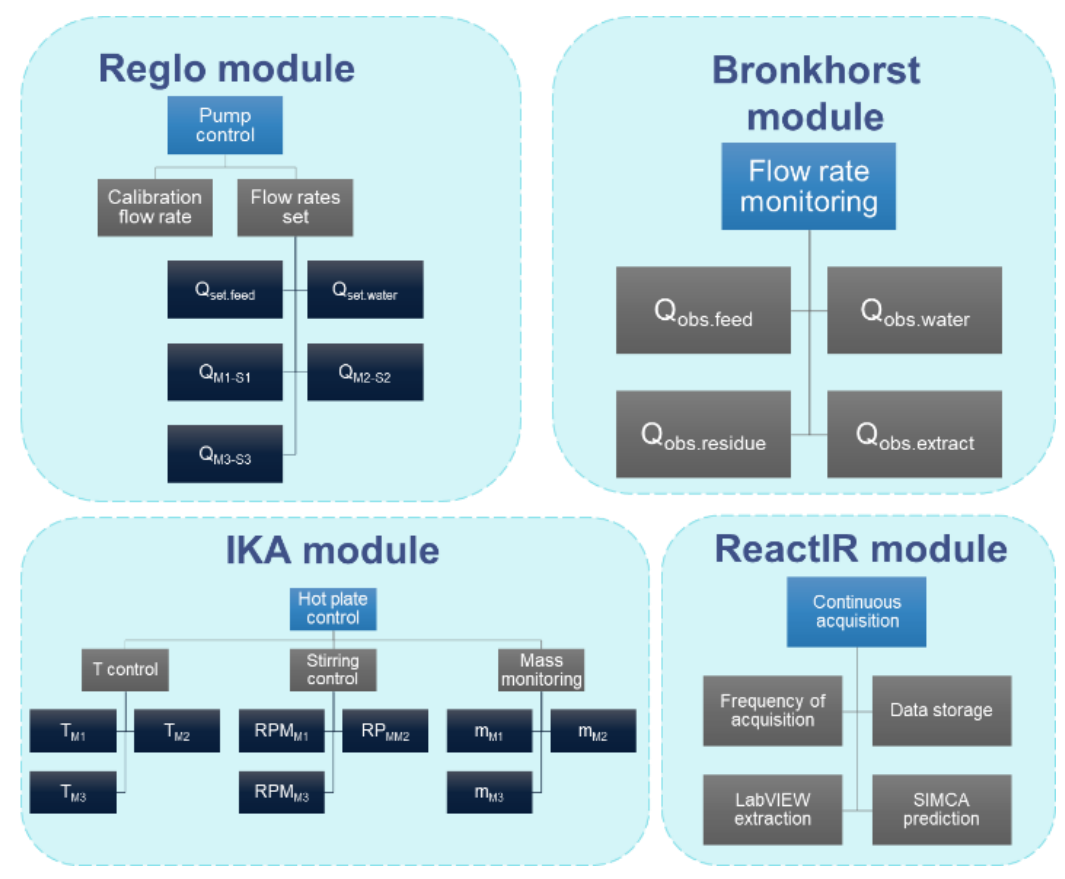

Graph 2. Four integrated modules for the automation of the 3-stages countercurrent liquid-liquid extraction.

\section{Results and discussion}

\section{1. $\quad$ PLS predictive performance for PAT}

The Partial Least Squares method was exploited to build a predictive model that is able to convert the IR spectra acquired by the PAT implemented in the automated platform. This step is vital for any further feedback loop of the automated extraction. It allowed to covert a graphical analytical spectrum to a quantitative monitoring of the concentration of the different ingredients at the downstream of the process. It is clear from Graph 3 that the PLS model performs optimally by including 3 PLS components. The variance explained by the generated reached $96 \%$ and the mean square error dropped down to 4 as illustrated graph 3.B.

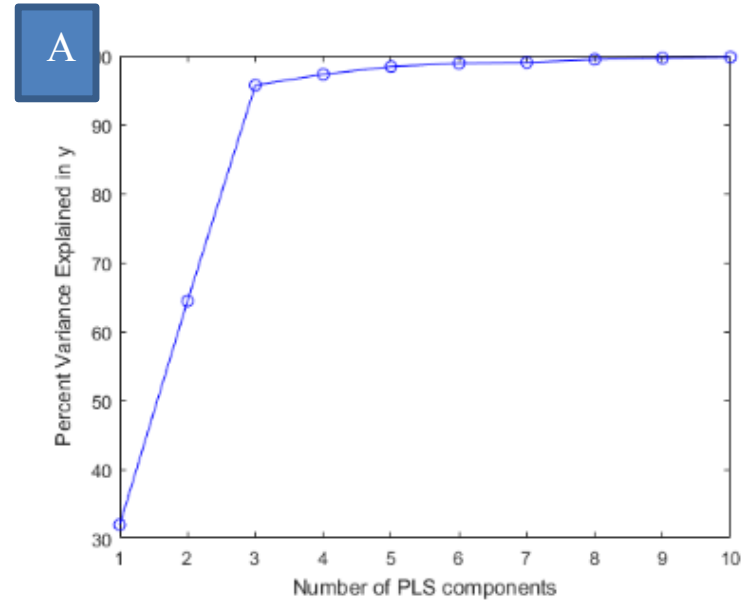

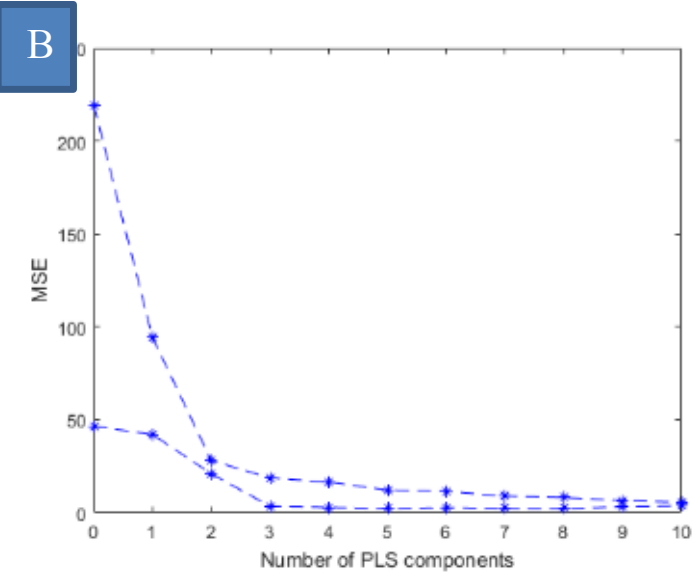

Graph 3: Predictive performance of the PLS model as a function of the number of considered components. A) Percentage of accumulated variance explained by the model. B) Mean square error.

\subsection{Dynamics of the automated 3-stages LLE platform}

The elucidation of the dynamics that drive the extraction in the 3 stage countercurrent liq-liq extract is important to conduct any further optimisation of the extraction factors in order to improve the purity of the product or to increase the yield of extract or to minimise the energy and the water required to carry out the extraction. The dynamics of the extraction was monitored by analysing periodically samples extracted from the downstream of the platform via HPLC separation and quantification. The Graph 4 shows the partitioning of trimetozine and the trimethoxybenzoic acid in extract and in the residue. Therefore, it was straightforward to evaluate the variation of these two analytes over time in both extract and residue. The platform achieved a steady state (i.e. A stationary phase 
illustrated by a plateau in the concentration of the analytes). As shown in Graph 4.B, the extraction platform reached the steady

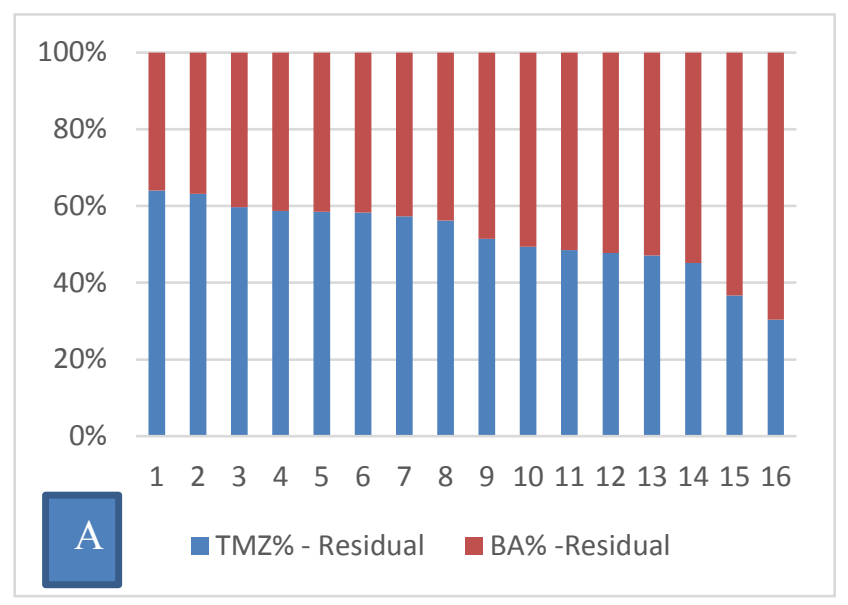

state from the $10^{\text {th }}$ sample which is occurring after $270 \mathrm{~min}$ from the start of the extraction.

Graph4: Percentage of extraction over time in the 3-stages liquid-liquid extraction platform. A) HPLC percentage of trimetozine and trimethoxybenzoic acid in the residue. B). HPLC percentage of trimetozine and trimethoxybenzoic acid in the extract.
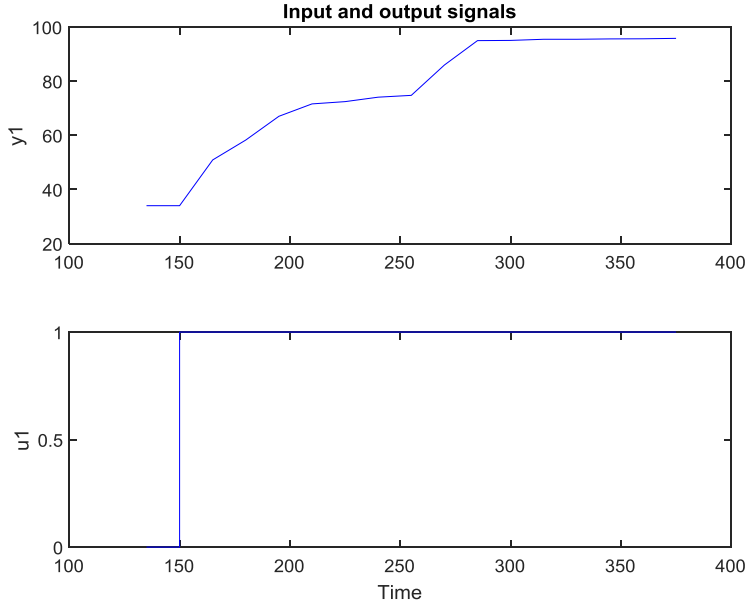

Graph 5: The dynamics of extraction over time. Y1 represents the yield of extraction and $t 1$ represents the flow rate of water in the platform.

The addition of water was triggered after $135 \mathrm{~min}$ from the beginning of extraction. The following sampling demonstrated the behaviour of the platform in terms of trimetozine extraction process. Water flow rate was stabilised at $1 \mathrm{ml} / \mathrm{min}$ over the whole process. The concentration of trimetozine kept increasing until achieving $95.04 \%$ after $285 \mathrm{~min}$ from the starting of the liquid flow within the platform.

The dynamics of extraction monitored in the liq-liq extraction platform was simulated using Simulink in MATLAB. Different regression models were generated in order to fit the experimental behaviour of extraction. The blue curve represents the initial fitting and the $5^{\text {th }}$ regression in pink represent the best fitting achieved by optimising the regression coefficients. Therefore, at tf5, $92.19 \%$ of fitting was achieved at tf5 to predict the dynamics of the extraction platform before reaching the steady-state.

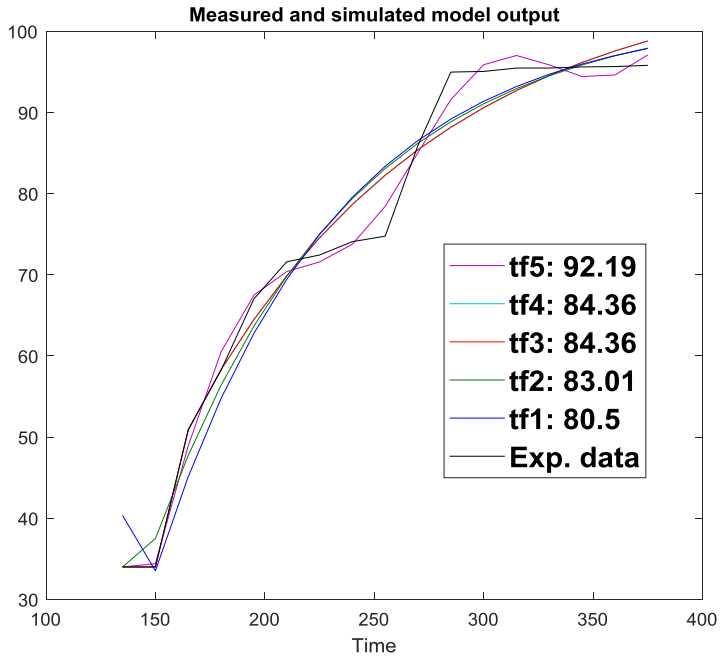

Graph 6: Five different models gathered from the black-box simulation of the extraction dynamics.

\section{4.}

\section{Conclusions}

A 3-stages liq-liq counter current extraction platform was implemented to conduct the extraction of trimetozine from a mixture containing mainly analyte of interest and the impurity (trimethoxybenzoic acid). The platform controlled and monitored the flow rates at the different streams of the platform and the PAT module allowed the monitoring of the extraction performance through an inline IR probe. A full evaluation of the partitioning of the analyte of interest was conducted by exploiting a PLS predictive model. The obtained concentrations of the extract and the impurity were employed to monitor the dynamics of the extraction and to build a black-box model to predict the extraction behaviour of trimetozine before reaching a steady-state regime. 


\section{AUTHOR INFORMATION}

\section{Corresponding Author}

* (Word Style "FA_Corresponding_Author_Footnote"). Give contact information for the author(s) to whom correspondence should be addressed.

\section{Present Addresses}

†If an author's address is different than the one given in the affiliation line, this information may be included here.

\section{Author Contributions}

The manuscript was written through contributions of all authors. / All authors have given approval to the final version of the manuscript. / \$These authors contributed equally. (match statement to author names with a symbol)

\section{Funding Sources}

Any funds used to support the research of the manuscript should be placed here (per journal style).

\section{Notes}

Any additional relevant notes should be placed here.

\section{ACKNOWLEDGMENT}

(Word Style "TD_Acknowledgments"). Generally, the last paragraph of the paper is the place to acknowledge people (dedications), places, and financing (you may state grant numbers and sponsors here). Follow the journal's guidelines on what to include in the Acknowledgement section.

\section{ABBREVIATIONS}

CCR2, CC chemokine receptor 2; CCL2, CC chemokine ligand 2; CCR5, CC chemokine receptor 5; TLC, thin layer chromatography.

\section{REFERENCES}

[1] M. Alexovič, B. Horstkotte, I. Šrámková, P. Solich, and J. Sabo, "Automation of dispersive liquid-liquid microextraction and related techniques. Approaches based on flow, batch, flow-batch and in-syringe modes," TrAC Trends Anal. Chem., vol. 86, pp. 39-55, Jan. 2017.

[2] N. Weeranoppanant et al., "Design of Multistage Counter-Current LiquidLiquid Extraction for Small-Scale Applications," Ind. Eng. Chem. Res., 2017.

[3] E. Boyaci et al., "Sample preparation with solid phase microextraction and exhaustive extraction approaches: Comparison for challenging cases," Analytica Chimica Acta. 2015.

[4] R. Jain and R. Singh, "Applications of dispersive liquid-liquid microextraction in forensic toxicology," TrAC - Trends in Analytical Chemistry. 2016

[5] M. Saraji and M. K. Boroujeni, "Recent developments in dispersive liquidliquid microextraction," Anal. Bioanal. Chem., 2013.

6] P. L. Kole, G. Venkatesh, J. Kotecha, and R. Sheshala, "Recent advances in sample preparation techniques for effective bioanalytical methods," Biomedical Chromatography. 2011

[7] E. B. Pinxterhuis, J. B. Gualtierotti, S. J. Wezenberg, J. G. de Vries, and B. L. Feringa, "Highly Efficient and Robust Enantioselective Liquid-Liquid Extraction of 1,2-Amino Alcohols utilizing VAPOL- and VANOL-based
Phosphoric Acid Hosts," ChemSusChem, 2018.

B. Schuur, B. J. V. Verkuijl, A. J. Minnaard, J. G. de Vries, H. J. Heeres, and B. L. Feringa, "Chiral separation by enantioselective liquid-liquid extraction," Org. Biomol. Chem., vol. 9, no. 1, pp. 36-51, 2011.

[9] K. Tang, Y. Wang, P. Zhang, Y. Huang, and G. Dai, "Process optimization of continuous liquid-liquid extraction in centrifugal contactor separators for separation of oxybutynin enantiomers," Sep. Purif. Technol., 2015.

[10] B. Schuur, B. J. V Verkuijl, A. J. Minnaard, J. G. de Vries, H. J. Heeres, and B. L. Feringa, "Chiral separation by enantioselective liquid-liquid extraction," Org. Biomol. Chem., vol. 9, no. 1, pp. 36-51, 2011.

[11] Y. Q. Tang and N. Weng, "Salting-out assisted liquid-liquid extraction for bioanalysis.," Bioanalysis, vol. 5, no. 12, pp. 1583-98, 2013.

[12] B. Peng, Y. Shen, Z. Gao, M. Zhou, Y. Ma, and S. Zhao, "Determination of total iron in water and foods by dispersive liquid-liquid microextraction coupled with microvolume UV-vis spectrophotometry," Food Chem., 2015.

[13] M. Tuzen and O. Z. Pekiner, "Ultrasound-assisted ionic liquid dispersive liquid-liquid microextraction combined with graphite furnace atomic absorption spectrometric for selenium speciation in foods and beverages," Food Chem., 2015.

[14] W. Ahmad, A. Al-Sibaai, A. S. Bashammakh, H. Alwael, and M. S. ElShahawi, "An ultrasound-assisted ion association dispersive liquid-liquid microextraction coupled with micro-volume spectrofluorimetry for chromium speciation," RSC Adv., 2016.

[15] C. I. C. Silvestre, J. L. M. Santos, J. L. F. C. Lima, and E. A. G. Zagatto, "Liquid-liquid extraction in flow analysis: A critical review," Anal. Chim. Acta, vol. 652, no. 1-2, pp. 54-65, Oct. 2009.

[16] "Chapter 10 Liquid-liquid extraction: Automation strategies," in Progress in Pharmaceutical and Biomedical Analysis, vol. 5, no. C, 2003, pp. 327 360

[17] F. Maya, J. M. Estela, and V. Cerdà, "Completely automated in-syringe dispersive liquid-liquid microextraction using solvents lighter than water," Anal. Bioanal. Chem., vol. 402, no. 3, pp. 1383-1388, Jan. 2012.

[18] C. S. M. Liew, X. Li, H. Zhang, and H. K. Lee, "A fully automated analytical platform integrating water sampling-miniscale-liquid-liquid extraction-full evaporation dynamic headspace concentration-gas chromatography-mass spectrometry for the analysis of ultraviolet filters," Anal. Chim. Acta, 2018.

[19] I. Domínguez, R. Romero González, F. J. Arrebola Liébanas, J. L. Martínez Vidal, and A. Garrido Frenich, "Automated and semi-automated extraction methods for GC-MS determination of pesticides in environmental samples," Trends in Environmental Analytical Chemistry. 2016.

[20] J. Zweigenbaum, K. Heinig, S. Steinborner, T. Wachs, and J. Henion, "High-Throughput Bioanalytical LC/MS/MS Determination of Benzodiazepines in Human Urine: 1000 Samples per 12 Hours," Anal. Chem., vol. 71, no. 13, pp. 2294-2300, 1999.

[21] S. L. C. Ferreira et al., "Box-Behnken design: An alternative for the optimization of analytical methods," Analytica Chimica Acta. 2007.

[22] M. Khajeh, "Response surface modelling of lead pre-concentration from food samples by miniaturised homogenous liquid-liquid solvent extraction: Box-Behnken design," Food Chem., vol. 129, no. 4, pp. 1832-1838, 2011.

[23] K. M. Khan et al., "Leishmanicidal potential of N-substituted morpholine derivatives: Synthesis and structure-activity relationships," Nat. Prod. Res., vol. 23, no. 5, pp. 479-484, 2009.

[24] I. Schmid and J. Aschoff, "A scalable software framework for data integration in bioprocess development," Eng. Life Sci., 2017.

[25] N. Hernández, R. Kiralj, M. M. C. Ferreira, and I. Talavera, "Critical comparative analysis, validation and interpretation of SVM and PLS regression models in a QSAR study on HIV-1 protease inhibitors," Chemom. Intell. Lab. Syst., vol. 98, no. 1, pp. 65-77, 2009.

[26] J. H. Moore, "Artificial intelligence programming with LabVIEW: genetic algorithms for instrumentation control and optimization," Comput. Methods Programs Biomed., vol. 47, no. 1, pp. 73-79, 1995. 
